\title{
The use of Nd:YAG laser in the treatment of hypertrophic venous lake of the upper lip
}

\begin{abstract}
Venous lake (VL) is a special case of venous malformation and is represented by an ectatic vein. Externally, this formation is manifested by a spot of blue color, soft consistency, which is emptied when pressed, but when you lower the finger again filled with blood. VL is typical for patients of the older age group. This malformation causes significant cosmetic discomfort and can be a source of bleeding, reducing the quality of life. Traditional VL treatment includes surgical removal, electrocoagulation, argon removal, cryodestruction, sclerotherapy. These treatments are not always effective in the treatment of VL, in addition, very often in the postoperative period, patients experience significant discomfort from pain, in some cases, a recurrence of the disease is possible. Laser treatment is devoid of such disadvantages. The use of Nd:YAG laser (1064nm) long pulse allows you to effectively remove VL without side effects. A clinical observation of hypertrophic upper lip VL using Nd:YAG laser $(1064 \mathrm{~nm})$ with a good clinical result is presented. The use of Nd:YAG lasers in the treatment of VL located on the lips is an effective and safe treatment method. For the procedure does not require long preparation, the procedure itself is performed quickly. This wavelength $(1064 \mathrm{~nm})$ penetrates the skin without damaging it. After the procedure, the risk of complications is very low.
\end{abstract}

Keywords: venous lake, venous malformation, laser, Nd:YAG
Volume 9 Issue 5 - 2019

\section{Safin DA, Romanov DV \\ Center of the vascular pathology, Russia}

Correspondence: Safin DA, pediatric surgeon, Center of the Vascular Pathology, Russia, Tel +7499-704-03-02, Email safindinar@ya.ru

Received: October 12, 2019 | Published: October 29, 2019
Abbreviations: ISSVA, International society for the study of vascular anomaly; laser, light amplification stimulated radiation radiation; Nd:YAG, neodymium-doped yttrium-aluminum garnet; KTP, potassium phosphate titanyl; PDL, pulse-dye laser

\section{Introduction}

According to the ISSVA classification, all vascular anomalies are divided into tumors and malformations. Malformations are divided into venous, lymphatic, capillary and arteriovenous malformations and fistulas. Venous lake (VL) is a special case of venous malformation, which has virtually no communication with the draining veins and is located superficially in the papillary layer of the dermis. ${ }^{1}$ This formation is often mistakenly called "hemangioma". VL was first described in 1956 by Bean and Walsh. ${ }^{2}$ This formation is typical for age-related patients. Clinically manifested by a flat spot of blue or purple color, located at the level of the skin or slightly rising above it. The size of the formation averages from 2 to $10 \mathrm{~mm} .{ }^{3}$ Under palpation it soft consistence, painless, under you click is emptied, but under omission finger again is filled with blood (color is returning). Microscopically, the VM is an expanded vein containing several channels and located in the papillary layer of the dermis. Most often located in the lips, face, neck and ear, that is, it is the area that most often can be damaged by the sun. In addition, it can be located on the mucous membrane of the cheeks, tongue or gums. Predisposing factors that cause the appearance of VL include: solar damage, chronic trauma, which cause damage to the adventitia of blood vessels or the occurrence of vascular thrombosis. ${ }^{4}$ This malformation causes significant cosmetic and psychological discomfort ${ }^{5}$ for the patient, in some cases there may be rapid growth, which is accompanied by the appearance of a defect of the skin over the formation and bleeding, which is accompanied by a pronounced pain syndrome.

Treatment of VL is diverse and includes surgical removal, electrocoagulation, argon removal, cryodestruction, sclerotherapy, various types of lasers and light exposure (diode laser, $\mathrm{CO}_{2}$ laser, argon, pulse dye laser, Nd:YAG, as well as a combination of different lasers). ${ }^{1,2,4,6}$ Almost all of the proposed methods remove the formation through an incision or destruction of tissue, which causes the formation of a skin defect, and on the mucous membrane such an injury heals for a long time and is usually accompanied by severe swelling, discomfort and in some cases pain. Treatment with Nd:YAG laser has its advantages the chromophore for a given wavelength of $1064 \mathrm{~nm}$ is carboxyhemoglobin, so the laser action penetrates the skin and does not damage it. That allows to influence only VL without occurrence of various complications. ${ }^{7}$ (Figure 1-3)

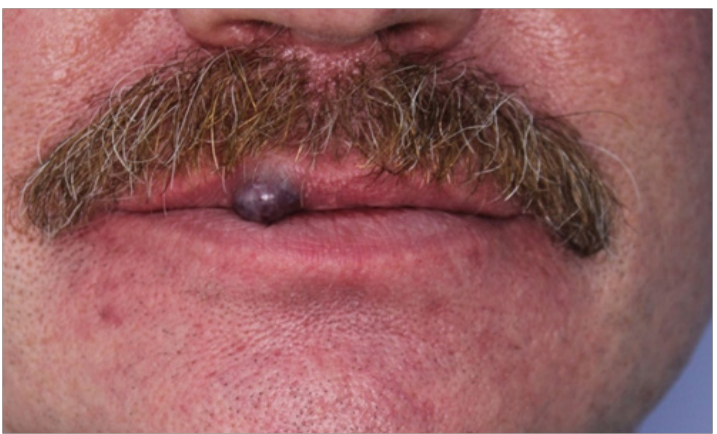

Figure I View ot the patient before treatment. 


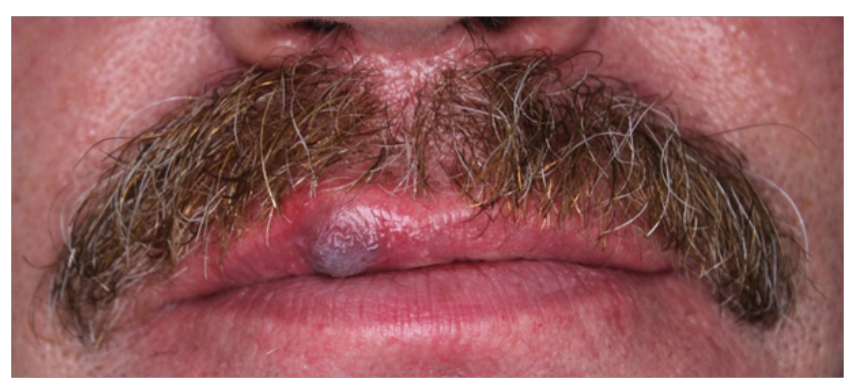

Figure 2 View ot the patient after first treatment Nd-YAG laser I064nm.

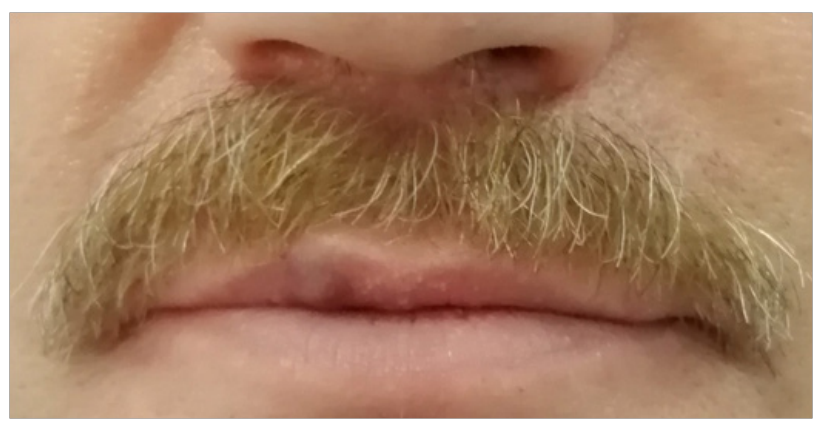

Figure 3 View of the patient after second treatment Nd-YAG laser I064nm.

\section{Materials and methods}

The result of treatment of a 55-year-old man with 3 Fitzpatrick phototype with hypertrophic VL on the upper lip (photo 1) is presented. This education in the patient for more than 30 years. It gradually increases in size and rises above the level of the skin. There was no trauma or bleeding. Previously, the patient did not seek help. After signing the informed consent, laser treatment sessions were performed. Local anesthesia before the procedure was not required. Performed 2 session of laser treatment Nd:YAG laser (Cutera Excel $\mathrm{V}$, Cutera, USA). In the first session, the following modes are used: power $70 \mathrm{j} / \mathrm{cm}^{2}$, pulse durations $45 \mathrm{~ms}$, in the second $90 \mathrm{j} / \mathrm{cm}^{2}$, pulse durations $50 \mathrm{~ms}$. The duration of the each procedure was less than 1 minute.

\section{Results}

After the laser exposure, there is a significant decrease in the formation, improvement of the color of the lip mucosa, due to this, the cosmetic result is significantly improved (photo 2,3 ). In the postoperative period, complications were not noted. Immediately after the procedure, the patient returned to work and led a normal lifestyle.

\section{Discussion}

Laser treatment unlike traditional surgery, sclerosis, cryodestruction, electrocoagulation is a more gentle treatment. Laser exposure is different from wavelength, so for example waves of 532nm (KTP), 585-595nm (PDL), 1064nm (Nd:YAG) do not damage the skin, unlike $800-810 \mathrm{~nm}$ (diode laser) or $10600 \mathrm{~nm}\left(\mathrm{CO}_{2}\right.$ laser).
The laser wavelength of $1064 \mathrm{~nm}$ has special properties-compared to other waves, it is able to penetrate deeper into the tissue before reaching the chromophore. The main chromophore is carboxyhemoglobin, which is contained in venous blood, so this type of laser is used to influence the venous component, for example, telangiectasia of blue/ purple color, reticular veins, etc. The chromophore for the wavelength of $532 \mathrm{~nm}$ and $585-595 \mathrm{~nm}$ is oxyhemoglobin, which is contained in arterial blood, so these waves are suitable for the treatment of capillary malformations (port wine stains), hemangiomas, telangiectasias of red color and others. The depth of penetration of Nd:YAG laser can be 6 $\mathrm{mm}$, and KTP and PDL only $1-2 \mathrm{~mm}$. It is these features of Nd:YAG laser can effectively act on various formations containing venous blood, such as VL.

\section{Conclusion}

The use of Nd:YAG lasers in the treatment of VL located on the lips is an effective and safe treatment method. For the procedure does not require long preparation, the procedure itself is performed quickly. This wavelength $(1064 \mathrm{~nm})$ penetrates the skin without damaging it. After the procedure, the risk of complications is very low.

\section{Acknowledgments}

None.

\section{Funding}

None.

\section{Conflict of interest}

There is no conflict of interest.

\section{References}

1. Mlacker S, Shah VV, Aldahan AS, et al. Laser and light-based treatments of venous lakes: a literature review. Lasers Med Sci. 2016;31(7):15111519 .

2. Cheung ST, Lanigan SW. Evaluation of the treatment of venous lakes with the 595-nm pulsed-dye laser a case series. Clin Exp Dermatol. 32(2):148-150.

3. Jing Y, Xiaohong G, Juan T. Treatment of venous lake with multiwavelength 595 and 1064nm lasers in Asian Fitzpatrick skin type IV patients. Photodermatol Photoimmunol Photomed. 2017;33(5):267270.

4. Voynov PP, Tomov GT, Mateva NG. Minimal Invasive Approach for Lips Venous Lake Treatment by $980 \mathrm{~nm}$ Diode Laser with Emphasis on the Aesthetic Results. capital A, Cyrillic Clinical Series. Folia Med (Plovdiv). 2016;58(2):101-107.

5. Menni S, Marconi M, Boccardi D, et al. Venous lakes of the lips: prevalence and associated factors. Acta Derm Venereol. 2014;94(1):7475.

6. Saverio C, Luisa L, Angela T, et al. Diode laser treatment of venous lake of the lip. Clin Case Rep. 2018;6(9):1923-1924.

7. Bekhor PS. Long-pulsed Nd:YAG laser treatment of venous lakes: report of a series of 34 cases. Dermatol Surg. 2006;32(9):1151-1154. 